Published in final edited form as:

Cancer Discov. 2018 March ; 8(3): 354-369. doi:10.1158/2159-8290.CD-17-0679.

\title{
Identifying and Targeting Sporadic Oncogenic Genetic Aberrations in Mouse Models of Triple Negative Breast Cancer
}

\author{
Hui Liu ${ }^{*}, 1$, Charles J. Murphy ${ }^{\star}, 2,7$, Florian A. Karreth ${ }^{3}$, Kristina B. Emdal ${ }^{4}$, Kangkang Yang ${ }^{1}$, \\ Forest M. White ${ }^{4}$, Olivier Elemento ${ }^{2}$, Alex Toker ${ }^{1,5}$, Gerburg M. Wulf ${ }^{6}$, and Lewis C. \\ Cantley ${ }^{7,8}$ \\ ${ }^{1}$ Department of Pathology, and Cancer Center, Beth Israel Deaconess Medical Center, Harvard \\ Medical School, Boston, MA 02115, USA \\ ${ }^{2}$ Institute for Computational Biomedicine, Weill Cornell Medical College, New York, NY 10021, \\ USA
}

${ }^{3}$ Department of Molecular Oncology, H. Lee Moffitt Cancer Center and Research Institute, Tampa, FL 33612, USA

${ }^{4}$ Department of Biological Engineering, Koch Institute for Integrative Cancer Research, MIT, Cambridge, MA 02139, USA

${ }^{5}$ Department of Pathology, and Cancer Center, Beth Israel Deaconess Medical Center, Harvard Medical School, and Ludwig Center at Harvard, Boston, MA 02115, USA

${ }^{6}$ Division of Hematology/Oncology, Beth Israel Deaconess Medical Center, Harvard Medical School, Boston, MA 02115, USA

${ }^{7}$ Meyer Cancer Center, Weill Cornell Medicine, New York, NY 10021, USA

${ }^{8}$ Meyer Cancer Center, Weill Cornell Medicine, New York, NY 10021, USA

\begin{abstract}
Triple negative breast cancers (TNBC) are genetically characterized by aberrations in TP53 and a low rate of activating point mutations in common oncogenes, rendering it challenging in applying targeted therapies. We performed whole exome sequencing (WES) and RNAseq to identify somatic genetic alterations in mouse models of TNBCs driven by loss of Trp53 alone or in combination with Brca1. Amplifications or translocations that resulted in elevated oncoprotein expressions or oncoprotein-containing fusions, respectively, as well as frame-shift mutations of tumor suppressors were identified in approximately $50 \%$ of the tumors evaluated. While the spectrum of sporadic genetic alterations was diverse, the majority had in common the ability to activate the MAPK/PI3K pathways. Importantly, we demonstrated that approved or experimental drugs efficiently induce tumor regression specifically in tumors harboring somatic aberrations of
\end{abstract}

Corresponding Author: Lewis C. Cantley, The Sandra and Edward Meyer Cancer Center, Weill Cornell Medicine, 413 East 69th St., 13th Floor, Box 50, New York, NY 10021. Phone: 646.962.6132; Fax: 646.962.0575; lcantley@ med.cornell.edu.

Contributed equally to this work.

Disclosure of Potential Conflicts of Interests: LCC is a founder and member of the BOD of Agios Pharmaceuticals and is a founder and receives research support from Petra Pharmaceuticals. These companies are developing novel therapies for cancer. No potential conflicts of interest were disclosed for other authors. 
the drug target. Our study suggests that the combination of WES and RNAseq on human TNBC will lead to the identification of actionable therapeutic targets for precision medicine guided TNBC treatment.

\section{Keywords}

Precision Medicine; Triple Negative Breast Cancer; Fusion kinase; FGFR2; RAF

\section{Introduction}

Targeted therapies for breast cancer treatment using estrogen receptor (ER)-antagonists, monoclonal antibodies or small molecules directed against HER2 for tumors expressing these cognate targets have resulted in great improvement in patient survival in $\mathrm{ER}^{+}, \mathrm{PR}^{+}$and HER2 amplified patient populations (1-3). By contrast, there are no similar specific targeted therapies available for triple negative breast cancer (TNBC), which is defined by the absence of ER, progesterone receptor (PR) and HER2. This disease occurs more commonly in younger women and in women of African and Hispanic descent, and patients are at higher risk of local or distant recurrence and worse prognosis when compared to other breast cancer subtypes (4). Despite significant efforts, little progress has been made, and chemotherapy remains the standard of care for TNBC patients.

TNBC patients exhibit highly variable responses to chemotherapy, which is likely linked to the significant molecular differences observed among these tumors (4). Recent profiling of the tumor transcriptomes have highlighted the heterogeneity in $\operatorname{TNBC}(5,6)$, and transcriptional profiling allowed the classification of TNBC into discrete subgroups, each with distinct expression profiles and, importantly, clinical implications (5,7). Large-scale whole exome sequencing (WES) and whole genome sequencing projects further demonstrated the molecular basis for TNBC tumor heterogeneity, revealing a diverse range and number of mutations, chromosomal number variations and translocations (7-9). While over $80 \%$ of TNBC patients harbor point mutations or deletions in the TP53 locus and a smaller subset have point mutations in genes controlling the PI3K pathway (PTEN, PIK3CA and INPP4B), gain of function point mutations that are common in other cancers such as those found in BRAF, KRAS and EGFR are rare events in TNBCs (8-11). Several of these oncogenic drivers are potentially targetable using small molecules that are either FDAapproved or currently undergoing clinical evaluation. However, the low frequency of each oncogenic mutation in TNBC patients precludes the broad usage of any single targeted agent in this patient population. Rather, these data provide a rationale for pursuing a precision medicine approach to identify potential drivers, and to tailor treatment regimens based upon the somatic alteration spectrum specific to each patient's tumor.

Several issues confound applying precision medicine approaches to TNBCs. Most trials do not require genomic profiling on biopsies at the time of enrollment, rendering correlations between clinical responses and genetic aberrations difficult to establish. In recent years, patient-derived-xenografts (PDXs) have been used to test personalized medicine approaches 
in treating patient-derived breast cancers (12). However, these models use immunocompromised mice and their clinical applicability remains unclear.

Here, we took a complementary approach to establish genetically engineered mouse models (GEMMs) harboring breast-specific Tp53 deletion with or without concomitant Brcal deletion. We carried out whole exome sequencing (WES) and RNAseq on the tumors that these TNBC models developed and confirmed that they faithfully recapitulated features of human TNBC disease. We were able to identify putative driver aberrations, including chromosomal amplifications and deletions, chromosomal translocations, mutations and proto-oncogene overexpression specific to individual tumors. Interestingly, although different tumors harbor different genetic aberrations, many of these share the ability of enhancing MAPK and PI3K pathway activation. In addition, when a particular tumor was treated with frontline therapeutic drugs targeting predicted oncogenic drivers specific to that tumor, sustained tumor remission was achieved. These data further support the tumorpromoting functions of these genetic events and support the paradigm of genomics-guided treatment in TNBC. Our results suggest that the combination of whole exome sequencing and RNAseq on human TNBC can reveal genetic aberrations that would be missed by conventional approaches that evaluate mutational events in panels of known oncogene. Importantly, our results suggest that this approach can significantly extend the use of approved or experimental drugs for TNBC patients who progress on standard of care treatments.

\section{Results}

\section{Establishment of Brca1-deficient and Brca1-WT TNBC mouse models}

The majority of TNBC tumors possess TP53 mutations and approximately $15 \%$ of patients carry germ-line BRCA1 mutations (4). We generated two cohorts of mice to model both BRCA1-germline mutation carriers and sporadic TNBCs: K14cre; $p 53^{\text {flox/flox }}$; Brcal $1^{\text {flox/flox }}$, defined as Brca1-deficient cohort; and K14cre; p53 flox/flox; Brcal wt/wt, defined as Brca1WT cohort. (Figure S1A). The majority of tumors developed from these mouse models were ER-, PR- and HER2-negative, with histopathological characteristics reminiscent of human TNBCs (Figure 1A, and S1B). To further investigate whether these mouse models resembled human TNBC, we performed RNAseq on RNAs isolated from tumors and from age-matched normal mammary glands to analyze their transcriptional profiles. Both AIMS and PAM50 classifier algorithms $(13,14)$, as well as unsupervised hierarchical clustering were used to identify the intrinsic subtypes of these mouse tumors. AIMS assigned almost all mouse tumors with high probability into basal-like breast cancer tumor subtypes, while all three normal mammary glands were assigned to the "normal-like" subtype (Figure 1B). Similarly, PAM50 assigned most of the tumors to the basal-like breast cancer subtype, which is the subtype most commonly associated with TNBC (15) (Figure S1C). In addition, unsupervised hierarchical clustering of the mouse tumors with RNAseq expression profiles from TCGA breast cancer samples on the 138 shared genes between mouse and human revealed that the mouse tumors segregated with human TNBCs, and not with other breast cancer subtypes (Figure S1D, Supplemental Table 1). Therefore, both immunohistological analysis and 
transcriptional profiling indicated that these mouse tumors, developed on a $T p 53$-deficient background with or without Brcal, provide reasonable models for human TNBC.

\section{RNAseq and WES revealed heterogeneous aberrations in murine TNBC}

To identify spontaneous genetic aberrations that potentially are oncogenic drivers of these tumors, we performed WES on tumor DNA and matched germ-line DNA. We observed low somatic mutation rates; an average of 30 somatic mutations (and 17 non-synonymous mutations) were found in the coding exons per tumor, ranging from 0 to 104 mutations across our set (Figure 1C and supplementary Table 2). Among all non-synonymous mutations, we only identified $\operatorname{Kras} \mathrm{Q} 61 \mathrm{H}$ and $\operatorname{Hras} \mathrm{Q} 61 \mathrm{~K}$ as potential oncogenic driver mutation in two of the primary tumors. Both mutations have been observed in human tumors and have been shown to drive transformation in cell lines $((16,17)$ and http:// cancer.sanger.ac.uk/cosmic/mutation/overview?id=554). Although other mutations were identified, they were not at hotspots in human oncogenes and their oncogenic potential remains to be determined (Figure S1E). We compared the mouse tumor mutational signatures to the 21 mutation signatures identified in human cancers (18), and found that Brca1-deficient tumors were most similar to signature 3, which is associated with BRCA1/2 mutations in human breast cancer (Figure 1D)(8). We also found that the mutation signature for Brca1-WT tumors were most similar to signature 17, which has an unknown etiology (Figure 1D and S1F).

Next, we analyzed somatic copy number alterations (CNAs) to investigate chromosomal gains and losses in these murine tumors. Overall, the CNA profiles exhibited marked variability among the tumors (Figure 1E and Supplementary Table 3). Consistent with human breast cancer (19), the Brcal-deficient subgroup exhibited more amplifications and deletions than the Brcal-WT subgroup (Figure S1G), supporting that loss of Brca1 contributes to genomic instability. The most recurrent events were focal amplifications on chromosome 6 centered on the Met locus and amplifications on chromosome 9 centered on the Yap1 locus (Figure 1F and Fig. S1H). Amplification of the Met gene was observed in 10 out of the 32 Brcal-WT primary tumors but none in Brcal-Deletion tumors. Amplifications in the Yap1 gene were found in 8/32 Brcal-WT and 1/30 Brcal-Deletion tumors. We also observed recurrent chromosomal gain at the $M y c$ locus on chromosome 15, and deletion of the $R b$ locus within a broad region on chromosomal 14 (Figure $\mathrm{S} 1 \mathrm{H}$ ). In addition to these recurrent amplification events, we identified sporadic high focal amplification on chromosome 7 that includes the Fgfr 2 locus, on chromosome 11 that includes Egfr locus, and a bi-allelic deletion on chromosome 19 that includes the Pten locus in one tumor (Figure $1 \mathrm{G}$ and S1I).

We integrated WES data with RNAseq data to investigate whether CNA alterations lead to changes in the expression levels of the affected genes. Among the recurrent amplifications, 7 of the 10 Met amplified tumors indeed showed increased Met transcript levels, and 6 of the 9 Yap1 amplifications resulted in elevated mRNA expression (Figure 1F). In addition, we found that tumor \#1 with the Fgfr2 amplification expressed significantly higher level of Fgfr 2 mRNA compared to other tumors that were CNA-neutral at this locus. The tumor with a focal amplification of Efgr was among the ones expressing the highest Egfr mRNA levels. 
The tumor harboring a bi-allelic Pten deletion expressed the lowest Pten mRNA levels among all tumors (Figure 1G). It has been shown that over-expression of MET (20,21), EGFR (22-24), YAP1 (25-27), or FGFR2 $(28,29)$, as well as deletion of PTEN $(30,31)$ contribute to TNBC tumorigenesis, and our data suggest that through combined RNAseq and WES data analyses, potential oncogenic drivers of TNBC tumors can be identified.

\section{Chromosomal rearrangements in primary and transplanted tumors}

Analysis of RNAseq data also revealed the presence of chromosomal translocation events, which varied from tumor to tumor (Figure S2A) and a subset of these rearrangements resulted in mRNA fusions (Figures 2A and 2B). Several of the fusions involved genes encoding proto-oncogenic protein kinases (Fig. S2B and Supplementary Table 4). Three different Ffgr2-fusions were observed, Fgfr2-Dnm3 (Dynamin 3) in tumor \#1 and Fgfr2Tns1 (Tensin 1) in tumor \#6, and Fgfr2-Zmynd8 where the distal portion of the Fgfr2 gene was replaced by the translocation partners, potentially generating fusion proteins (Figure 2C) with intact kinase domains. Proteins generated from these Fgfr2-fusion transcripts share the same domain architecture as FGFR2-fusions identified in human breast cancer $(32,33)$. Notably, in tumor \#1 where Fgfr2-Dnm3 translocation was observed, the chromosomal region encoding Fgfr2 was highly amplified, while in tumor \#6 with an Fgfr2-Tns 1 fusion, the chromosomal region covering Fgfr2 exhibited some gain in copy number (Figure S1I). Translocations involving the Raf family kinases were also detected, including a $D \lg 1$ (disc large 1)-B-Raf fusion in tumor \#3, Dhx9 (DEAH-Box Helicase 9)-Raf1 fusion in tumor \#5, and Rpl32 (Ribosomal Protein L32)-Raf1 fusion in tumor \#25. In all cases, the fusion proteins had an intact RAF kinase domain while the RAF N-terminal regulatory domain was replaced by the fusion partners, similar to BRAF-fusions identified in metastatic breast cancer (34) and other BRAF- and RAF1-fusions found in different human tumor types (3537) (Figure $2 \mathrm{C}$ and Figure $\mathrm{S} 2 \mathrm{C}$ ).

We validated the presence of fusion transcripts in tumors harboring Fgfr2-and Raf1-fusions by RT-PCR and traditional Sanger Sequencing using primer pairs that either match the input RNAs (match primers) or not (mismatch primers). PCR products of expected sizes were observed for matching RNA/primers, but not for mismatch RNA/primers (Figure 2D). When RT-PCR products were gel extracted and sequenced by traditional Sanger sequencing, the junctions were confirmed (Figure 2E, and S2D-F).

Thus, through combined WES and RNAseq analyses, we detected spontaneous chromosomal amplifications of Met, Yap1, Egfr and Fgfr2, a bi-allelic deletion of Pten, oncogenic mutations in Kras and Hras, as well as sporadic fusions of Fgfr2-Dnm3, Fgfr2Tns1, Fgfr2-Zmydn8, Dhx9-Raf1, and Rpl32-Rafland Dlg1-Braf in distinct tumors.

\section{Aberrant genetic events possess oncogenic activity by enhancing MAPK and/or PI3K pathway activations}

We next investigated whether the fused transcripts resulted in functional fusion proteins. We detected the Fgfr2-Dnm3 fusion protein in tumor \#1 at the predicted size of $99 \mathrm{kDa}$ using antibodies recognizing the N-terminus of Fgfr2 and the C-terminus of Dnm3. Importantly, the presence of Fgfr2-Dnm3 coincided with elevated phosphorylation of the Fgfr2 substrate 
Frs2 (Figure 3A and Figure S3A for sample key). To confirm the presence of Fgfr2-Tns1 fusion proteins, we immunoprecipitated Fgfr2 and subjected proteins at the predicted molecular mass ( $265 \mathrm{kDal}$ ) for mass spectrometry, and identified peptides from both Fgfr2 and Tns1 (Figure 3B and Figure S3B for peptide sequences matching Fgfr2 or Tns1). We also confirmed the presence of the Dhx9-Raf1 fusion protein in tumor \#5 at the expected molecular weight (51 kDa) (Figure 3C), and this tumor exhibited elevated activation of the MAPK signaling pathway as measured by ERK phosphorylation, compared to tumors that lacked Raf fusions (Figure 3C, and Figure S3C).

The tumor with high Met mRNA expression had elevated tyrosine-phosphorylated Met compared to tumors that lacked Met overexpression (Fig. 3D and Fig. S3D). The tumor with bi-allelic deletion of Pten (\#17) exhibited low levels of Pten protein and elevated PI3Kpathway activation detected by high levels of phospho-AKT and high levels of phosphorylation of the AKT substrate, PRAS40 (Figure 3E and S3E).

\section{Fgfr2-, Braf- and Raf1-Fusion kinases exhibit enhanced MAPK and/or PI3K pathway activation and oncogenic activity}

To evaluate the oncogenic potential of the novel fusion proteins, we cloned HA-tagged Fgfr2-Dnm3, Dhx 9-Raf1 and Rpl32-Raf1 into pBabe-puro and generated stable NIH3T3 cells and carried out signaling analyses and soft-agar colony formation assays. FGFR2 fusions, similar to the ones we observed in mice albeit with different translocation partners, have been reported in human breast and thyroid cancers $(32,33)$ and we included one of these fusions, FGFR2-CCDC6 (coiled-coil domain containing 6, Figure S4A) in our assays.

Expression of the fusion proteins was confirmed by blotting for the HA-tag, Fgfr2, and Raf1, (Figure S4B and S4C). We found that expression of Fgfr2-Dnm3 and FGFR2-CCDC6 resulted in FGFR2 activation detected by phospho-Tyr653/654 of FGFR2, and substrate phosphorylation detected by phospho-FRS2 at Tyr436. As a consequence, downstream PI3K activation was enhanced as measured by phospho-AKT (Figure 4A and Figure S4B, S4C). Moreover, both Fgfr2-Dnm3 and FGFR2-CCDC6 induced anchorage-independent growth of NIH3T3 cells in soft agar (Figure 4B). To understand the mechanisms for enhanced pathway activation, we transiently expressed epitope-tagged FGFR2-fusions in HEK293T cells and assayed for ligand-independent oligomerization/dimerization by co-immunoprecipitation in serum-free condition, and we found both FGFR2-CCDC6 and Ffgr2-Dnms3 undergo homooligomerization in the absence of FGF but fail to oligomerize with endogenous FGFR2s (Figure 4C). These results are consistent with a model in which the C-terminal fusion partner (CCDC6 and Dnms3) mediates the homo-multimerization.

By contrast, expression of Dhx9-Raf1 and Rpl32-Raf1 stimulated MAPK pathway activation with minimal impact on PI3K pathway activation in NIH3T3 cells (Figure 4D), and this also resulted in the significantly increased capacity to form colonies in soft agar (Figure 4E).

We also analyzed the effects of these fusion proteins in immortalized human mammary epithelial cells (HMECs). Consistent with the results obtained in NIH3T3 cells, Fgfr2-Dnm3 expression increased both MAPK and PI3K signaling in epithelial cells (Figure S4D). Surprisingly, Dhx9-Raf1 expression in epithelial cells also enhanced activation of the PI3K 
pathway (Figure S4D). Previous studies have shown autocrine production of various growth factors contribute to Raf1-mediated PI3K activation in mammary epithelial cells (38-41), which may explain the signaling differences observed between NIH3T3 cells and HMECs. In addition, expression of the fusion proteins resulted in increased proliferation of HMECs (Figure 4F). cMet-overexpression resulted in spontaneous cMET phosphorylation in the absence of growth factors and enhanced pAKT and pERK levels (Figure 4G), as well as increased cell proliferation (Figure S4E).

Altogether, our results identify a likely driver event in approximately $50 \%$ of the 72 tumors evaluated (Figure $4 \mathrm{H}$ ). Our data show that the majority of the genetic alterations we identified have in common the ability to enhance activation of MAPK and/or PI3K signaling.

\section{Tumors harboring Fgfr2 fusion proteins are responsive to FGFR2-inhibition in vivo, resulting in complete tumor regression}

Next, we asked whether the murine TNBC tumors that spontaneously acquired the Fgfr 2 or Raf1 translocations would respond to drugs that target these protein kinases. To this end, we transplanted mouse TNBC tumors with identifiable potential genetic drivers in cohorts of nude mice, and each mouse received a designated treatment regimen (Figure S5A). Targeted agents were selected based on (i) high specificity and high competence indicated by low $\mathrm{IC}_{50}$, (ii) good in vivo bioavailability with low in vivo dosing, and (iii) approved for cancer therapy or currently in phase II or III clinical trials. Based on these criteria, NVP-BGJ398 was chosen as an FGFR-inhibitor (Figure S5B), Trametinib (GSK1120212) as a MEKinhibitor (Figure S6A), and Crizotinib as a MET-inhibitor (Figure S6D) for in vivo treatments.

For tumor \#1, which is Brca1-deficient with a spontaneous Fgfr2-Dnm3 translocation, we evaluated the efficacy of FGFR-inhibitor BGJ398 alone, or in combination with Olaparib (a PARP-inhibitor approved for BRCA1 mutant cancers) (Figure 5A), anticipating that tumors may become resistant on monotherapy. We also included Crizotinib in this trial, reasoning that Met is not the driver in this particular tumor and it should therefore not respond to this treatment. We determined that the dose of BGJ398 needed to suppress Frs2 phosphorylation was $30 \mathrm{mg} / \mathrm{kg}$ (Figure 5B), which is estimated to be equivalent to a human dose of $2.4 \mathrm{mg} / \mathrm{kg}$ (42), and the dose of Olaparib had previously been reported (43). We did not observe general toxicity over prolonged treatment (Figure 5C). At this dose, BGJ398 alone was sufficient to induce complete tumor regression (Figure 5D). This outcome was a significant improvement compared to a pan-PI3K inhibitor BKM120 treatment, which only resulted in slower rate of tumor progression (Figure S5C). Importantly, Crizotinib had no effect on the growth of tumor \#1 (Figure 5D), although it was capable of delaying tumor progression when Met levels are elevated (Figure 6F), supporting the notion that the choice of treatment should be based on the genetics of the tumor. At early time points (up to 33 days), BGJ398 and the combination of BGJ398 and Olaparib were equally effective in inducing tumor remission. However, three of six tumors on BGJ398 monotherapy developed resistance, and on average at day 43 (day 36, 47 and 57) tumors relapsed to the initial volume (Figure 5E). By contrast, tumors treated with BGJ398 and Olaparib combination therapy did not relapse when the 
experiment ended, and all tumors reached complete response (CR) (Figure 5E). Using a second PARP-inhibitor BioMarine BMN673 (44) in combination with BGJ398, no tumor relapse was observed for up to 80 days (Figure S5D). Our data suggest that spontaneous Fgfr2-Dnm3 fusions can drive tumorigenesis in the breast.

For tumor \#6, which is Brca1-WT with a spontaneous Fgfr2-Tns1 translocation, we compared BGJ398 alone to the PI3K-inhibitor BKM120 (Figure S5E) since Brca1-WT murine tumors are less sensitive to PARP-inhibition relative to PI3K-inhibition (Figure S5E). We treated tumor-bearing mice with BGJ398, BKM120 alone, or in combination (Figure S5F). We observed that BKM120 treatment resulted in stable disease (SD), although with general toxicity (Figure S5G). However, BGJ398 alone induced tumor regression, and the combination of both drugs caused fast and complete tumor remission until no tumor tissue could be detected (Figure 5F). The combination did not cause further toxicity beyond what was elicited by BKM120 alone (Figure S5G). Our data show that spontaneous tumors with Fgfr 2 aberrations are responsive to FGFR2-inhibitor treatments, suggesting complete tumor regression can be achieved when genetic alterations of each tumor are carefully considered.

\section{Tumors with Raf1-fusion or overexpressing Met responded to specific inhibitors targeting these pathways}

Since MEK inhibitors have been shown to effectively treat human tumors driven by mutated Raf family members, we treated tumor \# 5 that expresses the Dhx9-Raf1fusion protein with the MEK inhibitor Trametinib (GSK1120212). A 3mg/kg/day dose of Trametinib was effective in blocking Erk phosphorylation (Figure 6A). Mice implanted with tumor \#5 were given Trametinib as either a single agent, or in combination with Olaparib since tumor \#5 is a Brca1-deficient tumor (Figure 6B). Either single agent or combination therapy did not cause general toxicity at the doses used (Figure S6B). Consistent with our hypothesis, Trametinib alone significantly delayed tumor growth, with an initial phase of stable disease, which transitioned to progressive disease after 14 days (Figure S6C). Olaparib alone initially did not halt tumor growth, but slowly resulted in tumor regression. When mice were treated with a combination of both the MEK and PARP inhibitors, tumor \#5 completely regressed without relapse upon extended treatment for up to 50 days till the experiment ended (Figure 6C). It is also important to note that when treated with the FGFR-inhibitor BGJ398, tumor \#5 (Dhx9-Raf1 fusion) failed to respond (Figure 6C and S6C), further supporting the importance of identifying and suppressing specific oncogenic activities in specific patient subpopulations.

RNAseq revealed that a group of tumors derived from the same primary tumor (Tumor \#2) (Figure 1F) exhibited approximately a 30-fold increase in cMet mRNA levels compared to other tumors evaluated, despite no amplification in the Met locus. Since Met overexpression enhances spontaneous MET activation in cells, we evaluated the effect of Crizotinib, an inhibitor of both MET and ALK tyrosine kinases, on the growth of these tumors. At $50 \mathrm{mg} / \mathrm{kg}$, Crizotinib completely blocked cMET phosphorylation in tumors, as well as downstream AKT, ERK and S6 phosphorylation (Figure 6D) without general toxicity (Figure S6D). While overall survival for mice receiving control treatment is only 8 days, 
Crizotinib treatment delays tumor progression and tripled survival to 24 days (Figure 6E). Combining Crizotinib with BKM120 (Figure 6F) achieved stable disease in this particularly aggressive tumor (Figure 6E). The effect of Crizotinib on the tumor was probably a consequence of inhibiting MET since, as discussed above, Crizotinib had little effect on Tumor \#1 (Fig. 5D), which does not overexpress MET.

The results presented in Figures 5 and 6 indicate that either complete tumor regression or stable disease can be achieved, using monotherapy or combination therapy with inhibitors that target the pathways that are affected by the genetic aberrations specific to individual tumors. These in vivo treatment results not only confirm the functional importance of these genetic events in tumor development, but also provide evidence that individualized treatment design based on genomic information can lead to significantly improved outcomes.

\section{Human TNBCs harbor a broad range of targetable genetic alterations}

Our data show that despite the use of highly inbred mice and common initiating events (breast-specific deletion of $T p 53$ alone or deletion of both $T p 53$ and Brca1), tumors took diverse evolutionary pathways to become TNBC. Yet the majority of the tumors converged on genetic alterations enhancing MAPK and PI3K signaling pathways. Therefore, we analyzed TCGA human breast cancer data to investigate genetic alterations known to enhance MAPK and/or PI3K pathway activation.

We first interrogated 82 tumors in the TCGA database that were annotated as TNBC. Specifically, we searched for mutations and amplifications in $K R A S, B R A F$ and $R A F 1$ genes; mutation, amplification and overexpression in genes encoding for receptor tyrosine kinases (RTKs); as well as genes involved in the PI3K pathway. We found that approximately $90 \%$ of all TNBCs have at least one such genetic aberration (Figure 7A and Figure S7A). To determine whether this high prevalence is specific to TNBC, or is generally true for all breast cancer, we investigated the TCGA breast cancer database (816 cases) and the METABRIC database (Molecular Taxonomy of Breast Cancer International Consortium, 2509 cases), and found that $72 \%$ and $56 \%$ of breast tumor samples show at least one of these changes, with the majority of the tumors displaying two or more alterations (Figure 7A and S7B). The result from this search suggests that while genetic changes leading to potential MAPK/PI3K activation are common, such alterations may be particularly important for TNBC development.

Since mouse tumors with spontaneous Fgfr-signaling pathway activation are sensitive to the FGFR-inhibitor BGJ398, we were interested in further investigating genetic alterations of the FGFR-family of receptor tyrosine kinases and their ligands. In addition to translocations, mutations and focal amplifications can also activate the FGFR family $(32,33,45,46)$. Therefore, we also analyzed the COSMIC databases as well as TCGA and METABRIC databases to identify mutations for FGFR and FGF family members. We found that chromosomal amplifications of the FGFR1, FGFR2 and FGF3/4/19 loci (Figure 7B and S7C) resulted in increased mRNA levels of FGFR1, FGFR2 and FGF4/19 (Figure 7C and S7D). Overall, $34 \%$ and $26 \%$ of all breast cancer patients showed FGFR1, FGFR 2 and/or FGF3 mutation/amplification/overexpression in TCGA and METABRIC, respectively (Figure S7E). Mutations in FGFR1 are rare, but $1.2 \%$ of all breast cancer patients have 
FGFR2 mutations, including known activating mutations such as $\mathrm{S} 252 \mathrm{~W}, \mathrm{~N} 549 \mathrm{~K}$ and K659E (Figure 7D).

These data indicate that in both genetically engineered mouse models and in human patients, the genetic alterations in TNBC are quite diverse but converge on a relatively limited number of ways to activate the MAPK and/or PI3K pathways (Figure 7E).

\section{Discussion}

In this work, we generated two GEMM models that are $T p 53$-deficient, with or without Brcal-deficiency, and carried out whole exome sequencing and RNAseq on these spontaneously developed tumors. We found that these TNBC mouse models recapitulate many aspects of human TNBC, including the overall transcriptional profile, mutational burden and signature as well as heterogeneous CNAs. We identified spontaneous genetic aberrations that were likely drivers of the evolution of these tumors. These genetic aberrations include both recurrent chromosomal amplifications of the Met and Yap1 loci, and sporadic amplification at the Fgfr 2 and Egfr loci, a bi-allelic deletion of Pten in individual tumors. In addition, spontaneous Fgfr2-Dnm3, Fgfr2-Tns1, Fgfr2-Zymnd8, Dhx 9-Raf, Rpl32-Raf1 and Dlg1-BRaf translocations that generate overexpressed, and/or constitutively active protein kinases were also discovered as oncogenic drivers. Point mutations of known oncogenes were rare; however, we identified tumors with activating mutations in Kras and Hras. Our results also showed that the majority of these genetic aberrations have in common the ability to enhance MAPK and PI3K pathway signaling. Next we asked to what extent these pathways are activated by genetic alterations known to occur in human breast cancer. We searched TCGA breast cancer database for KRAS, BRAF, and $R A F 1$ mutations and amplifications, RTK gene mutations, amplifications and overexpressions, YAP1 amplification/gain/overexpression, PIK3CA mutations and amplifications, as well as PTEN and INPP4B deletions, all of which can potentially activate the MAPK and/or PI3K pathways. More than $60 \%$ of breast tumors bear at least one of these aberrations, while over $90 \%$ of the TNBC subgroup of breast cancers exhibit these genetic lesions, suggesting the importance of MAPK and PI3K signaling pathway activation in TNBC tumorigenesis.

Through combined WES and RNAseq analyses, we identified potential oncogenic drivers in approximately $50 \%$ of tumors against which frontline drugs are also available

(Supplementary Table 5). It is worth noting that almost all of these genetic driver fusions would not be detected by conventional targeted sequencing approaches that only investigate mutational events in panels of known oncogenes. The mutational burden of murine tumors is relatively low, averaging 30 somatic mutations per tumor with 17 non-synonymous mutations. These numbers are comparatively lower than those of human TNBC, which average 127 somatic mutations per tumor with 107 being non-synonymous (TCGA data analysis and (9)), reflecting cross-species/age differences. Nevertheless, we do observe similar mutational patterns between Brca1-deficient murine tumors and Brca1-mutant human breast tumors (Figure 1). 
We found recurrent Met and Yap amplification in 20\% and 22\% of primary mouse tumors investigated, as well as individual tumors showing increased mRNA levels without chromosomal amplification. Overall, approximately $25 \%$ of the primary tumors show amplification/gain/overexpression of Met or Yap (Figure 1 and S1). Our data are consistent with human TCGA data, where $27 \%$ or $29 \%$ of TNBC patients show amplification/gain/ overexpression of $M E T$ or $Y A P$, supporting the importance of these genetic events in TNBC development $(20,21,27)$. Current data also point to the role of YAP1 in activation of the PI3K/MAPK pathway via increased PIK3CB, HRAS or GAB2 expression, or increased IRS phosphorylation in diverse types of tumors (47-50). We also observed recurrent c- $M y c$ amplification and $R b 1$ deletion in the mouse tumors, although both tend to occur in broader chromosomal regions. These data are consistent with a report by Holstege in mouse tumors (19), and also CBIO Portal analyses of human TNBC (http://www.cbioportal.org/index.do? session_id=59edd065498e5df2e29710eb\&show_samples=false\&). In addition to these recurrent CNAs, sporadic amplifications and deletions that affect cancer-related genes were identified, including high amplification of Fgfr 2 and Egfr loci, and deep deletion of the Pten locus, as well as shallow amplification of the Cdk6, Aurka, and Jun loci, and deletion of the Nf1 loci (Supplementary Table \# 3). Our data suggest that although normal mouse and human chromosomes bear major differences in their architectures, mouse tumors developed over 7-12 months exhibited similar changes in their cognate regions to human tumor genomes.

In addition to chromosomal amplifications and deletions at known oncogenic loci, chromosomal translocations were found to be the oncogenic drivers in a subset of the murine tumors. Interestingly, although mutational burden is low in human breast cancer, ranking $20^{\text {th }}$ among 30 different cancer types (18), the chromosomal rearrangement frequency ranks among the highest (Figure S7F and data generated based on "TCGA Fusion gene Data Portal" http://54.84.12.177/PanCanFusV2/), although the frequency of recurrent translocations is low. Traditionally viewed as important oncogenic drivers for hematological cancers (51), chromosomal rearrangement events have been identified and increasingly recognized as potent drivers in human solid tumors (33,34,36,52-54). Through next generation sequencing, a number of gene fusions with low frequency were identified in breast cancer patients, including FGFR2, FGFR3, BRAF, NTRK3, and MET $(32,33,36,53-$ 55). We also identified chromosomal translocations involving Fgfr2, Braf and Raf1 that resulted in constitutively active fusion kinases in the mouse tumors. We noticed that the fusion partners in the mouse tumors are different from those found in the human counterparts. However, it has been shown that these kinases tend to fuse with different partners in human tumors, while maintaining similar domain architecture. Typically, fusion events involving FGFR2 and FGFR3 occur at the C-terminus, replacing the C-terminus of FGFRs with different fusion partners that provide the ability of the fused protein to undergo spontaneous homo-multimerization (Figure S7G) $(33,45,54)$. Our data confirmed that the fusion partner found in mouse tumors has similar functions (Figure 4C). Similarly it is commonly observed that a diverse set of genes can fuse with Raf family members, generating fusion kinases where the N-terminal regulatory domain of Raf is replaced by the fusion partner, resulting in constitutive activation of the Raf family kinases (Figure $\mathrm{S} 7 \mathrm{H}$ ) (34-37,53). 
Further evidence for the oncogenic function of a genetic alteration is provided when such alterations confer sensitivity to targeted therapy that results in an objective response only in patients harboring the genetic alteration. This, in fact, represents the litmus test for the precision medicine approach in cancer treatment. We carried out individualized treatment studies based on the specific driver(s) present in a particular tumor. This allowed us to confirm that these predicted drivers indeed are important for tumor growth in vivo. We show that two tumors with Fgfr2 fusions responded to the FGFR inhibitor BGJ398, a tumor with a Raf1 fusion responded to the MEK-inhibitor Trametinib and a tumor with overexpressed MET responded to the MET inhibitor Crizotinib, verifying that these spontaneous genetic aberrations were driving tumor growth in each of the individual tumors. Importantly, the tumor with Fgfr2-Dnm3 fusion did not respond to Met inhibition, nor was the FGFR2inhibitior BGJ398 effective on the Met overexpressing tumor, highlighting the importance of matching therapeutic drugs with oncogenic drivers.

Our study indicates that although the evolutionary courses that lead to breast tumor formation in the context of Tp53 deletion, with or without Brcal deletion, are diverse, they have in common the ability to activate the MAPK and/or PI3K pathway. However, targeting both PI3K and MAPK pathways resulted in high toxicity and is not a viable option for patients. Our study suggests approved drugs or experimental drugs could be effective therapies when targeting upstream driver events, which can be identified by combined WES and RNAseq efforts.

One such upstream driver event is the FGFR activation. The importance of FGFR pathway in tumorigenesis has increasingly been recognized $(56,57)$, and FGFR fusions have been found in bladder, thyroid, prostate, lung cancer, glioblastoma and cholangiocarcinomas (Figure S7H) $(33,45,54)$. We demonstrated that mouse Fgfr2-Dnm3 and human FGFR2CCDC6 fusion kinases share similar oncogenic potential and activation mechanism. Our result that the FGFR-inhibitor BGJ398 (58) is effective in treating murine tumors harboring Fgfr2-fusions is also consistent with recent data obtained in a cholangiocarcinomas PDX model harboring FGFR2-CCDC6 fusion treated with BGJ398 (59). Together these results support ongoing clinical trails targeting the FGFR in various cancers (https:// clinicaltrials.gov), and some of which have shown therapeutic benefits. A phase II trial evaluating the efficacy of the TKI258 FGFR-inhibitor in patients with or without FGFR2 mutations showed increased overall survival when mutations are detected (20.2 months with mutations vs 9.3 months without) (Clinical Trial \# NCT01379534). A recently published basket trial targeting patients with potential FGFR pathway activations also found BGJ398 provided disease control in $37 \%$ of patients ( 49 of 132 patients) who have failed all available standard therapies. And for breast cancer patients with FGFR1/2 amplification or mutation, BGJ398 alone resulted in stable disease in 31\% (10 of 31) patients, although the disease eventually progressed on this treatment (60). These data support the potential of FGFR inhibitors in cancer treatment, but also highlights their limitation as monotherapy in longterm disease management.

Similar to available human trials, we found that although tumors harboring FGFR2 fusions responded well initially to BGJ398 monotherapy, this response is not sustained and tumors relapsed despite continuous treatment (60). Given our success with the FGFR inhibitor 
BGJ398 as part of combination therapy with PARP inhibitor in treating Brca1-deficient tumors, or with BKM120 in treating Brca1-WT tumors, we suggest that including BGJ398 as part of combination treatment regimens warrants further investigation in patients with FGFR family genetic aberrations and either germline or sporadic defects in DNA repair pathways.

Fusion kinases involving the RAF family members (B-RAF and RAF1) have been found with high frequency in pilocytic astrocytomas $(61,62)$ and at low frequency in melanoma(63,64), pancreatic(35), prostate(37), thyroid (65)and metastatic breast cancer $(34,66)$. We reasoned that although RAF1-fusions have not been found in human TNBC, Dhx9-Raf1 identified in the murine models share the same architecture as fusionRAF1s discovered in other tumor types. Therefore, it is important to consider and evaluate fusion kinases involving the Raf family members as therapeutic targets in vivo, and identify effective treatments for these fusion-containing tumors in breast cancer and beyond. We reported here that a TNBC tumor with Raf1-fusion kinase is responsive to Trametinib as a single agent, and note that since this tumor was Brcal-deficient the efficacy of Trametinib was improved when combined with Olaparib. Our data suggest the potential of treating RAF1 fusion containing tumors with MEK inhibitors.

Given that $c M e t$ overexpression synergizes with $T p 53$-loss to induce basal-like breast cancer (typically TNBCs) $(20,21)$, and cMet amplification and overexpression correlate with TNBC, it is encouraging that the murine tumor with Met overexpression responded to the MET-inhibitor Crizotinib either as monotherapy or in combination with the PI3K-inhibitor BKM120. This result is consistent with reports that targeting MET in MET-amplified TNBC mouse tumors results in an initial complete response (67). Combining MET and PARP inhibitor has been shown to suppressed Olaparib-resistant TNBC tumor cell growth in vivo (68).

Another potential therapeutic target in TNBC that emerged from this study is YAP1, a transcription factor that is normally suppressed by the Hippo tumor suppressor pathway. YAP1 collaborates with other transcription factors including TEAD and AP1 to promote tumorigenesis and metastasis $(26,69,70)$. In fact, Verteporfin, which inhibits YAP1 and TEAD interaction and thus transcriptional activity, has been used in vitro and in vivo $(49,71)$. It will be interesting to investigate the efficacy of this approach in our TNBC mouse model.

In summary, we have generated mouse models that recapitulate human TNBC. Through combined WES and RNAseq, we identified amplifications and fusions of oncogenic drivers that would have been missed by evaluating mutations in panels of known oncogenes. We investigated the efficacy of this precision medicine approach by treating each tumor as an individual patient using frontline drugs targeting specific oncogenic drivers. Our results indicate the importance of performing RNAseq and whole exome sequencing on TNBC in order to identify the genetic aberrations driving the disease. These data also underscore the importance of combination therapy in order to elicit prolonged treatment efficacy. This work provides a proof-of-principle evidence for on-going basket trials, NCI-MATCH (NCT02465060), My Pathway Trial (NCT02091141) and the European ESMART 
(NCT02813135), which are designed to match the specific abnormalities with therapeutic drugs.

\section{Methods}

\section{Endogenous tumor generation}

All animal studies were reviewed and approved by the Institutional Animal Care and Use Committee (IACUC) at the Beth Israel Deaconess Medical Center. K14cre; Brcal ${ }^{\text {flox/flox }}$; $T p 53^{\text {flox/flox }}$ mice were obtained from Dr. Jos Jonkers' lab (Netherlands Cancer Institute).

\section{Orthotopic tumor implantation}

Tumor pieces were cut into $2 \mathrm{~mm}$ in diameter and inserted into the $4^{\text {th }}$ mammary fat pad of 8 -week old recipient mice via a $0.5 \mathrm{~cm}$ incision in the skin and the skin was closed with VetBond as described (43).

\section{Tumor treatment and tumor measurement}

Once tumors reached $8 \mathrm{~mm}$ in diameter as measured by electrical caliper (Fisher Scientific), mice were treated with indicated drugs obtained from MedChemExpress, LLC. For oral gavage, 100ul of drug suspension was administrated daily for six consecutive days, followed by one drug holiday. Tumor sizes were measured twice a week (length and width), and tumor volume was calculated as (3.14*length*width*width/6).

\section{RNA and library preparation}

Total RNA was prepared following the protocol for Promega ReliaPrep RNA Tissue Miniprep System (Z6111), and RNA integrity and concentration were measured using the Agilent 2100 Bioanalyzer (Agilent Technologies). cDNA libraries were prepared from 1535 ng RNA starting material (RIN values >6.0), using the TruSeq RNA Sample Preparation Kit (Illumina) according to the manufacturer's instructions, and quality was checked on an Agilent 2100 Bioanalyzer (Agilent Technologies). Sequencing was carried out on the HiSeq 2500 (Illumina) using paired end clustering and $51 \times 2$ cycles sequencing.

\section{Genomic DNA and library preparation}

Genomic DNA from tumor or liver samples was prepared following the protocol for Promega ReliaPrep Tissue DNA Miniprep System (A2051). SureSelect or NimbleGen Mouse exome capture kits were used to generate DNA library according to manufacturer's instructions. Sequencing was carried out using HiSeq4000 (Illunima) using paired end clustering and $51 \times 2$ cycles sequencing. The NCBI BioProject accession number for both RNAseq and WES sequences reported in this paper is PRJNA398328.

\section{Sequence Analysis}

The quality of the raw FASTQ files was checked with FastQC. RNAseq reads were mapped to GRCm38 using STAR and expression levels (FPKMs) quantified using Cufflinks with default parameters. WES reads were mapped to GRCm38 using BWA then de-duplicated, realigned around indels, and base recalibrated. We classified the mouse tumors into the 
breast cancer intrinsic subtypes using AIMs and PAM50 classifiers after conversion of mouse gene symbols to human Entrez gene IDs and human gene symbols, respectively. PAM50 centroids were re-computed using RNAseq expression profiles from TCGA breast cancer data and their associated, published PAM50 classification as gold standard. Gene fusions were called using FusionCatcher and filtered for false positives.

\section{Mutation and copy number analysis}

Somatic mutations were identified upon removing any mutations found in any tail, liver or normal mammary control samples, in mouse dbSNP, or with insufficient coverage in the control samples. Mutations were annotated with SnpEff. Copy number variants were called using CNVkit after removing low-quality reads. Sample-specific thresholds were computed to call amplifications and deletions.

\section{Cell culture}

Primary human mammary epithelial cells (HMECs) were isolated and immortalized on passage 3 as described (72) upon informed consent (DFHCC-IRB legacy 04-405). Cells were maintained in MEGM medium (Lonza, Walkersville, MD) and used within 10 passages. NIH3T3 cells were obtained from and authenticated by ATCC in 2014 and cultured in DMEM medium with $10 \%$ fetal calf serum and were used within 10 passages. Retrovirus and lentivirus preparation and infection were carried out as described (73) using Lipofectamine 2000 (ThermoFisher).

\section{Protein lysis, western Blots, immunoprecipitation (IP), silver staining and mass spectrometry (MS)}

Tumors and cells were lysed in RIPA lysis buffer with protease inhibitors and phosphatase inhibitors (Sigma), and protein quantifications were performed using BCA Protein Assay (Pierce). Equal amount of total proteins were used for Western blots or IP, and gels were stained using Pierce Silver Staining kit (\# 24612), while MS was carried out as described (74). See supplementary material and methods for detailed antibody information.

\section{Cell Proliferation and soft agar formation assay}

For cell proliferation assay, 1500 cells were plated in 96-well plates and measured with CellTiter-Glo (Promega, G7572). For soft agar colony assay, 10,000 cells were resuspended in $0.4 \%$ agar (SeaPlaque low melting agar, Lonza) in cell growth medium and plated on $0.8 \%$ agar in 6-well plates. Medium was changed every week and after 3 weeks, cells were fixed and stained in $0.005 \%$ Crystal Violet in $20 \%$ Methanol in PBS.

\section{Supplementary Material}

Refer to Web version on PubMed Central for supplementary material.

\section{Acknowledgments}

We thank Kangkang Yang for excellent technical support, Drs. Jos Jonkers, Takehiko Sasaki and Michael Begley for providing critical reagents, members of the Cantley, Wulf and Toker labs for suggestions, and Dr. Xiang and her 
team at the Genomics and Epigenomics Core Facility of Weill Cornell Medical College for next generation sequencing.

Financial Support: This work was supported by grants from the NIH R35 CA197588 (LCC), R01 GM041890 (LCC), PSOC U54 CA210184 (LCC), U24 CA210989 (OE), R01 CA194547 (OE), P50CA211024 (OE), 5P50 CA168504-03 (SPORE) (GMW), the Breast Cancer Research Foundation awards BCRF-16-021 (LCC) and BCRF-17-174 (GMW), the Jon and Mindy Gray Foundation (LCC), "Research supported by a Stand Up to Cancer Colorectal Cancer Dream Team Translational Research Grant (Grant Number: SU2C-AACR-DT22-17). Stand Up to Cancer is a program of the Entertainment Industry Foundation. Research grants are administered by the American Association for Cancer Research, the scientific partner of SU2C (LCC) a Susan Komen postdoctoral fellowship (HL), the Ludwig Center at Harvard (GMW, AT), The Mary Kay Ash Foundation (GMW), The Breast Cancer Alliance (GMW, LCC), DFCI Mens Initiative to Cure Womens Cancers (GMW), and a Novo Nordisk STAR postdoctoral fellowship (KBE).

\section{References}

1. Arteaga CL, Sliwkowski MX, Osborne CK, Perez EA, Puglisi F, Gianni L. Treatment of HER2positive breast cancer: current status and future perspectives. Nat Rev Clin Oncol. 2011; 9:16-32. [PubMed: 22124364]

2. Cardoso F, Costa A, Norton L, Cameron D, Cufer T, Fallowfield L, et al. 1st International consensus guidelines for advanced breast cancer (ABC 1). Breast. 2012; 21:242-52. [PubMed: 22425534]

3. Lin NU, Winer EP. Advances in adjuvant endocrine therapy for postmenopausal women. J Clin Oncol. 2008; 26:798-805. [PubMed: 18258989]

4. Bianchini G, Balko JM, Mayer IA, Sanders ME, Gianni L. Triple-negative breast cancer: challenges and opportunities of a heterogeneous disease. Nat Rev Clin Oncol. 2016; 13:674-90. [PubMed: 27184417]

5. Lehmann BD, Bauer JA, Chen X, Sanders ME, Chakravarthy AB, Shyr Y, et al. Identification of human triple-negative breast cancer subtypes and preclinical models for selection of targeted therapies. J Clin Invest. 2011; 121:2750-67. [PubMed: 21633166]

6. Perou CM, Sorlie T, Eisen MB, van de Rijn M, Jeffrey SS, Rees CA, et al. Molecular portraits of human breast tumours. Nature. 2000; 406:747-52. [PubMed: 10963602]

7. Curtis C, Shah SP, Chin SF, Turashvili G, Rueda OM, Dunning MJ, et al. The genomic and transcriptomic architecture of 2,000 breast tumours reveals novel subgroups. Nature. 2012; 486:346-52. [PubMed: 22522925]

8. Nik-Zainal S, Davies H, Staaf J, Ramakrishna M, Glodzik D, Zou X, et al. Landscape of somatic mutations in 560 breast cancer whole-genome sequences. Nature. 2016; 534:47-54. [PubMed: 27135926]

9. Shah SP, Roth A, Goya R, Oloumi A, Ha G, Zhao Y, et al. The clonal and mutational evolution spectrum of primary triple-negative breast cancers. Nature. 2012; 486:395-9. [PubMed: 22495314]

10. Gewinner C, Wang ZC, Richardson A, Teruya-Feldstein J, Etemadmoghadam D, Bowtell D, et al. Evidence that inositol polyphosphate 4-phosphatase type II is a tumor suppressor that inhibits PI3K signaling. Cancer Cell. 2009; 16:115-25. [PubMed: 19647222]

11. Stephens PJ, Tarpey PS, Davies H, Van Loo P, Greenman C, Wedge DC, et al. The landscape of cancer genes and mutational processes in breast cancer. Nature. 2012; 486:400-4. [PubMed: 22722201]

12. Byrne AT, Alferez DG, Amant F, Annibali D, Arribas J, Biankin AV, et al. Interrogating open issues in cancer precision medicine with patient-derived xenografts. Nat Rev Cancer. 2017; 17:254-68. [PubMed: 28104906]

13. Paquet ER, Hallett MT. Absolute assignment of breast cancer intrinsic molecular subtype. J Natl Cancer Inst. 2014; 107:357. [PubMed: 25479802]

14. Parker JS, Mullins M, Cheang MC, Leung S, Voduc D, Vickery T, et al. Supervised risk predictor of breast cancer based on intrinsic subtypes. J Clin Oncol. 2009; 27:1160-7. [PubMed: 19204204]

15. Foulkes WD, Smith IE, Reis-Filho JS. Triple-negative breast cancer. N Engl J Med. 2010; 363:1938-48. [PubMed: 21067385] 
16. Chang MT, Asthana S, Gao SP, Lee BH, Chapman JS, Kandoth C, et al. Identifying recurrent mutations in cancer reveals widespread lineage diversity and mutational specificity. Nat Biotechnol. 2016; 34:155-63. [PubMed: 26619011]

17. Misale S, Yaeger R, Hobor S, Scala E, Janakiraman M, Liska D, et al. Emergence of KRAS mutations and acquired resistance to anti-EGFR therapy in colorectal cancer. Nature. 2012; 486:532-6. [PubMed: 22722830]

18. Alexandrov LB, Nik-Zainal S, Wedge DC, Aparicio SA, Behjati S, Biankin AV, et al. Signatures of mutational processes in human cancer. Nature. 2013; 500:415-21. [PubMed: 23945592]

19. Holstege H, van Beers E, Velds A, Liu X, Joosse SA, Klarenbeek S, et al. Cross-species comparison of aCGH data from mouse and human BRCA1- and BRCA2-mutated breast cancers. BMC Cancer. 2010; 10:455. [PubMed: 20735817]

20. Graveel CR, DeGroot JD, Su Y, Koeman J, Dykema K, Leung S, et al. Met induces diverse mammary carcinomas in mice and is associated with human basal breast cancer. Proc Natl Acad Sci U S A. 2009; 106:12909-14. [PubMed: 19567831]

21. Ponzo MG, Lesurf R, Petkiewicz S, O’Malley FP, Pinnaduwage D, Andrulis IL, et al. Met induces mammary tumors with diverse histologies and is associated with poor outcome and human basal breast cancer. Proc Natl Acad Sci U S A. 2009; 106:12903-8. [PubMed: 19617568]

22. Brandt R, Eisenbrandt R, Leenders F, Zschiesche W, Binas B, Juergensen C, et al. Mammary gland specific hEGF receptor transgene expression induces neoplasia and inhibits differentiation. Oncogene. 2000; 19:2129-37. [PubMed: 10815804]

23. Marozkina NV, Stiefel SM, Frierson HF Jr, Parsons SJ. MMTV-EGF receptor transgene promotes preneoplastic conversion of multiple steroid hormone-responsive tissues. J Cell Biochem. 2008; 103:2010-8. [PubMed: 17960555]

24. Masuda H, Zhang D, Bartholomeusz C, Doihara H, Hortobagyi GN, Ueno NT. Role of epidermal growth factor receptor in breast cancer. Breast Cancer Res Treat. 2012; 136:331-45. [PubMed: 23073759]

25. Chang SS, Yamaguchi H, Xia W, Lim SO, Khotskaya Y, Wu Y, et al. Aurora A kinase activates YAP signaling in triple-negative breast cancer. Oncogene. 2016; 36:1265-75. [PubMed: 27593935]

26. Yu FX, Zhao B, Guan KL. Hippo Pathway in Organ Size Control, Tissue Homeostasis, and Cancer. Cell. 2015; 163:811-28. [PubMed: 26544935]

27. Zanconato F, Cordenonsi M, Piccolo S. YAP/TAZ at the Roots of Cancer. Cancer Cell. 2016; 29:783-803. [PubMed: 27300434]

28. Easton DF, Pooley KA, Dunning AM, Pharoah PD, Thompson D, Ballinger DG, et al. Genomewide association study identifies novel breast cancer susceptibility loci. Nature. 2007; 447:108793. [PubMed: 17529967]

29. Katoh M. FGFR inhibitors: Effects on cancer cells, tumor microenvironment and whole-body homeostasis (Review). Int J Mol Med. 2016; 38:3-15. [PubMed: 27245147]

30. Liu JC, Voisin V, Wang S, Wang DY, Jones RA, Datti A, et al. Combined deletion of Pten and p53 in mammary epithelium accelerates triple-negative breast cancer with dependency on eEF2K. EMBO Mol Med. 2014; 6:1542-60. [PubMed: 25330770]

31. Wang S, Liu JC, Kim D, Datti A, Zacksenhaus E. Targeted Pten deletion plus p53-R270H mutation in mouse mammary epithelium induces aggressive claudin-low and basal-like breast cancer. Breast Cancer Res. 2016; 18:9. [PubMed: 26781438]

32. Parker BC, Engels M, Annala M, Zhang W. Emergence of FGFR family gene fusions as therapeutic targets in a wide spectrum of solid tumours. J Pathol. 2014; 232:4-15. [PubMed: 24588013]

33. Wu YM, Su F, Kalyana-Sundaram S, Khazanov N, Ateeq B, Cao X, et al. Identification of targetable FGFR gene fusions in diverse cancers. Cancer Discov. 2013; 3:636-47. [PubMed: 23558953]

34. Ross JS, Wang K, Chmielecki J, Gay L, Johnson A, Chudnovsky J, et al. The distribution of BRAF gene fusions in solid tumors and response to targeted therapy. Int J Cancer. 2015; 138:881-90. [PubMed: 26314551] 
35. Chmielecki J, Hutchinson KE, Frampton GM, Chalmers ZR, Johnson A, Shi C, et al. Comprehensive genomic profiling of pancreatic acinar cell carcinomas identifies recurrent RAF fusions and frequent inactivation of DNA repair genes. Cancer Discov. 2014; 4:1398-405. [PubMed: 25266736]

36. Kumar-Sinha C, Kalyana-Sundaram S, Chinnaiyan AM. Landscape of gene fusions in epithelial cancers: seq and ye shall find. Genome Med. 2015; 7:129. [PubMed: 26684754]

37. Palanisamy N, Ateeq B, Kalyana-Sundaram S, Pflueger D, Ramnarayanan K, Shankar S, et al. Rearrangements of the RAF kinase pathway in prostate cancer, gastric cancer and melanoma. Nat Med. 2010; 16:793-8. [PubMed: 20526349]

38. de Larco JE, Todaro GJ. Growth factors from murine sarcoma virus-transformed cells. Proc Natl Acad Sci U S A. 1978; 75:4001-5. [PubMed: 211512]

39. McCarthy SA, Samuels ML, Pritchard CA, Abraham JA, McMahon M. Rapid induction of heparin-binding epidermal growth factor/diphtheria toxin receptor expression by Raf and Ras oncogenes. Genes Dev. 1995; 9:1953-64. [PubMed: 7649477]

40. Schulze A, Lehmann K, Jefferies HB, McMahon M, Downward J. Analysis of the transcriptional program induced by Raf in epithelial cells. Genes Dev. 2001; 15:981-94. [PubMed: 11316792]

41. Siriwardana G, Bradford A, Coy D, Zeitler P. Autocrine/paracrine regulation of breast cancer cell proliferation by growth hormone releasing hormone via Ras, Raf, and mitogen-activated protein kinase. Mol Endocrinol. 2006; 20:2010-9. [PubMed: 16613992]

42. Nair AB, Jacob S. A simple practice guide for dose conversion between animals and human. J Basic Clin Pharm. 2016; 7:27-31. [PubMed: 27057123]

43. Juvekar A, Burga LN, Hu H, Lunsford EP, Ibrahim YH, Balmana J, et al. Combining a PI3K inhibitor with a PARP inhibitor provides an effective therapy for BRCA1-related breast cancer. Cancer Discov. 2012; 2:1048-63. [PubMed: 22915751]

44. Shen Y, Rehman FL, Feng Y, Boshuizen J, Bajrami I, Elliott R, et al. BMN 673, a novel and highly potent PARP1/2 inhibitor for the treatment of human cancers with DNA repair deficiency. Clin Cancer Res. 2013; 19:5003-15. [PubMed: 23881923]

45. Gallo LH, Nelson KN, Meyer AN, Donoghue DJ. Functions of Fibroblast Growth Factor Receptors in cancer defined by novel translocations and mutations. Cytokine Growth Factor Rev. 2015; 26:425-49. [PubMed: 26003532]

46. Helsten T, Elkin S, Arthur E, Tomson BN, Carter J, Kurzrock R. The FGFR Landscape in Cancer: Analysis of 4,853 Tumors by Next-Generation Sequencing. Clin Cancer Res. 2015; 22:259-67. [PubMed: 26373574]

47. Garcia-Rendueles ME, Ricarte-Filho JC, Untch BR, Landa I, Knauf JA, Voza F, et al. NF2 Loss Promotes Oncogenic RAS-Induced Thyroid Cancers via YAP-Dependent Transactivation of RAS Proteins and Sensitizes Them to MEK Inhibition. Cancer Discov. 2015; 5:1178-93. [PubMed: 26359368]

48. Lin Z, Zhou P, von Gise A, Gu F, Ma Q, Chen J, et al. Pi3kcb links Hippo-YAP and PI3K-AKT signaling pathways to promote cardiomyocyte proliferation and survival. Circ Res. 2015; 116:3545. [PubMed: 25249570]

49. Wang C, Gu C, Jeong KJ, Zhang D, Guo W, Lu Y, et al. YAP/TAZ-Mediated Upregulation of GAB2 Leads to Increased Sensitivity to Growth Factor-Induced Activation of the PI3K Pathway. Cancer Res. 2017; 77:1637-48. [PubMed: 28202507]

50. Wang C, Jeong K, Jiang H, Guo W, Gu C, Lu Y, et al. YAP/TAZ regulates the insulin signaling via IRS1/2 in endometrial cancer. Am J Cancer Res. 2016; 6:996-1010. [PubMed: 27293994]

51. Wong S, Witte ON. The BCR-ABL story: bench to bedside and back. Annu Rev Immunol. 2004; 22:247-306. [PubMed: 15032571]

52. Shaver TM, Lehmann BD, Beeler JS, Li CI, Li Z, Jin H, et al. Diverse, Biologically Relevant, and Targetable Gene Rearrangements in Triple-Negative Breast Cancer and Other Malignancies. Cancer Res. 2016; 76:4850-60. [PubMed: 27231203]

53. Stransky N, Cerami E, Schalm S, Kim JL, Lengauer C. The landscape of kinase fusions in cancer. Nat Commun. 2014; 5:4846. [PubMed: 25204415] 
54. Yoshihara K, Wang Q, Torres-Garcia W, Zheng S, Vegesna R, Kim H, et al. The landscape and therapeutic relevance of cancer-associated transcript fusions. Oncogene. 2015; 34:4845-54. [PubMed: 25500544]

55. Kawazu M, Kojima S, Ueno T, Totoki Y, Nakamura H, Kunita A, et al. Integrative analysis of genomic alterations in triple-negative breast cancer in association with homologous recombination deficiency. PLoS Genet. 2017; 13:e1006853. [PubMed: 28636652]

56. Andre F, Cortes J. Rationale for targeting fibroblast growth factor receptor signaling in breast cancer. Breast Cancer Res Treat. 2015; 150:1-8. [PubMed: 25677745]

57. Tiseo M, Gelsomino F, Alfieri R, Cavazzoni A, Bozzetti C, De Giorgi AM, et al. FGFR as potential target in the treatment of squamous non small cell lung cancer. Cancer Treat Rev. 2015; 41:52739. [PubMed: 25959741]

58. Guagnano V, Furet P, Spanka C, Bordas V, Le Douget M, Stamm C, et al. Discovery of 3-(2,6dichloro-3,5-dimethoxy-phenyl)-1-\{6-[4-(4-ethyl-piperazin-1-yl)-phenylamin o]-pyrimidin-4yl \}-1-methyl-urea (NVP-BGJ398), a potent and selective inhibitor of the fibroblast growth factor receptor family of receptor tyrosine kinase. J Med Chem. 2011; 54:7066-83. [PubMed: 21936542]

59. Wang Y, Ding X, Wang S, Moser CD, Shaleh HM, Mohamed EA, et al. Antitumor effect of FGFR inhibitors on a novel cholangiocarcinoma patient derived xenograft mouse model endogenously expressing an FGFR2-CCDC6 fusion protein. Cancer Lett. 2016; 380:163-73. [PubMed: 27216979]

60. Nogova L, Sequist LV, Perez Garcia JM, Andre F, Delord JP, Hidalgo M, et al. Evaluation of BGJ398, a Fibroblast Growth Factor Receptor 1-3 Kinase Inhibitor, in Patients With Advanced Solid Tumors Harboring Genetic Alterations in Fibroblast Growth Factor Receptors: Results of a Global Phase I, Dose-Escalation and Dose-Expansion Study. J Clin Oncol. 2017; 35:157-65. [PubMed: 27870574]

61. Chen YH, Gutmann DH. The molecular and cell biology of pediatric low-grade gliomas. Oncogene. 2013; 33:2019-26. [PubMed: 23624918]

62. Jones DT, Kocialkowski S, Liu L, Pearson DM, Ichimura K, Collins VP. Oncogenic RAF1 rearrangement and a novel BRAF mutation as alternatives to KIAA1549:BRAF fusion in activating the MAPK pathway in pilocytic astrocytoma. Oncogene. 2009; 28:2119-23. [PubMed: 19363522]

63. Botton T, Yeh I, Nelson T, Vemula SS, Sparatta A, Garrido MC, et al. Recurrent BRAF kinase fusions in melanocytic tumors offer an opportunity for targeted therapy. Pigment Cell Melanoma Res. 2013; 26:845-51. [PubMed: 23890088]

64. Hutchinson KE, Lipson D, Stephens PJ, Otto G, Lehmann BD, Lyle PL, et al. BRAF fusions define a distinct molecular subset of melanomas with potential sensitivity to MEK inhibition. Clin Cancer Res. 2013; 19:6696-702. [PubMed: 24345920]

65. Cordioli MI, Moraes L, Carvalheira G, Sisdelli L, Alves MT, Delcelo R, et al. AGK-BRAF gene fusion is a recurrent event in sporadic pediatric thyroid carcinoma. Cancer Med. 2016; 5:1535-41. [PubMed: 27037835]

66. Craig DW, O'Shaughnessy JA, Kiefer JA, Aldrich J, Sinari S, Moses TM, et al. Genome and transcriptome sequencing in prospective metastatic triple-negative breast cancer uncovers therapeutic vulnerabilities. Mol Cancer Ther. 2012; 12:104-16. [PubMed: 23171949]

67. Pfefferle AD, Agrawal YN, Koboldt DC, Kanchi KL, Herschkowitz JI, Mardis ER, et al. Genomic profiling of murine mammary tumors identifies potential personalized drug targets for p53deficient mammary cancers. Dis Model Mech. 2016; 9:749-57. [PubMed: 27149990]

68. Du Y, Yamaguchi H, Wei Y, Hsu JL, Wang HL, Hsu YH, et al. Blocking c-Met-mediated PARP1 phosphorylation enhances anti-tumor effects of PARP inhibitors. Nat Med. 2016; 22:194-201. [PubMed: 26779812]

69. Lamar JM, Stern P, Liu H, Schindler JW, Jiang ZG, Hynes RO. The Hippo pathway target, YAP, promotes metastasis through its TEAD-interaction domain. Proc Natl Acad Sci U S A. 2012; 109:E2441-50. [PubMed: 22891335]

70. Zanconato F, Forcato M, Battilana G, Azzolin L, Quaranta E, Bodega B, et al. Genome-wide association between YAP/TAZ/TEAD and AP-1 at enhancers drives oncogenic growth. Nat Cell Biol. 2015; 17:1218-27. [PubMed: 26258633] 
71. Valero V 3rd, Pawlik TM, Anders RA. Emerging role of Hpo signaling and YAP in hepatocellular carcinoma. J Hepatocell Carcinoma. 2015; 2:69-78. [PubMed: 27508196]

72. Burga LN, Tung NM, Troyan SL, Bostina M, Konstantinopoulos PA, Fountzilas H, et al. Altered proliferation and differentiation properties of primary mammary epithelial cells from BRCA1 mutation carriers. Cancer Res. 2009; 69:1273-8. [PubMed: 19190334]

73. Liu H, Ong SE, Badu-Nkansah K, Schindler J, White FM, Hynes RO. CUB-domain-containing protein 1 (CDCP1) activates Src to promote melanoma metastasis. Proc Natl Acad Sci U S A. 2011; 108:1379-84. [PubMed: 21220330]

74. Sarma K, Cifuentes-Rojas C, Ergun A, Del Rosario A, Jeon Y, White F, et al. ATRX Directs Binding of PRC2 to Xist RNA and Polycomb Targets. Cell. 2014; 159:1228. [PubMed: 28898627] 


\section{Significance}

Using combined whole exome sequencing and RNAseq analyses, we identified sporadic oncogenic events in TNBC mouse models that share the capacity to activate the MAPK and/or PI3K pathways. Our data support a treatment tailored to the genetics of individual tumors that parallels the approaches being investigated in the ongoing NCI-MATCH, My Pathway Trial and the ESMART clinical trials. 
A
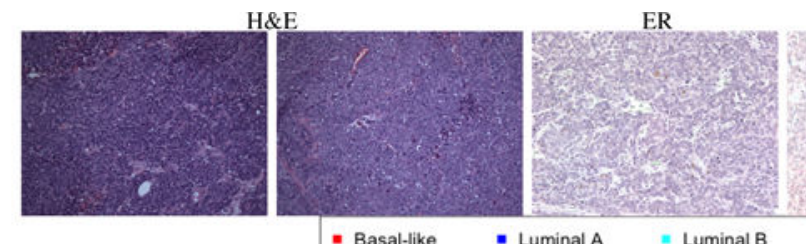

PR HER2

B

- Basal-like

Luminal A

" Luminal B

- HER2-enriched | Normal-like

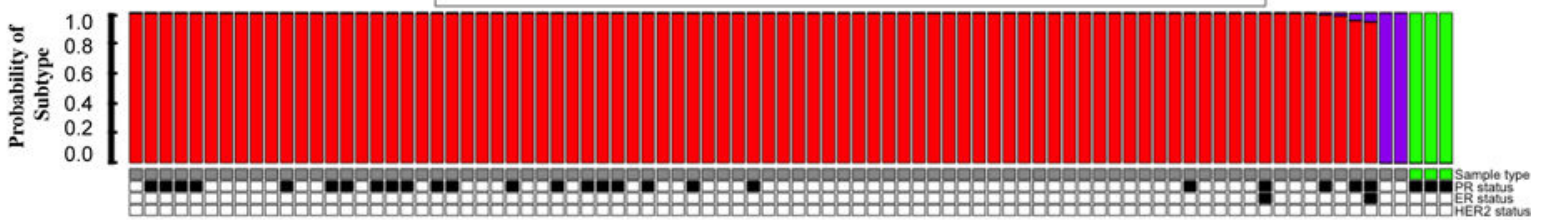

C

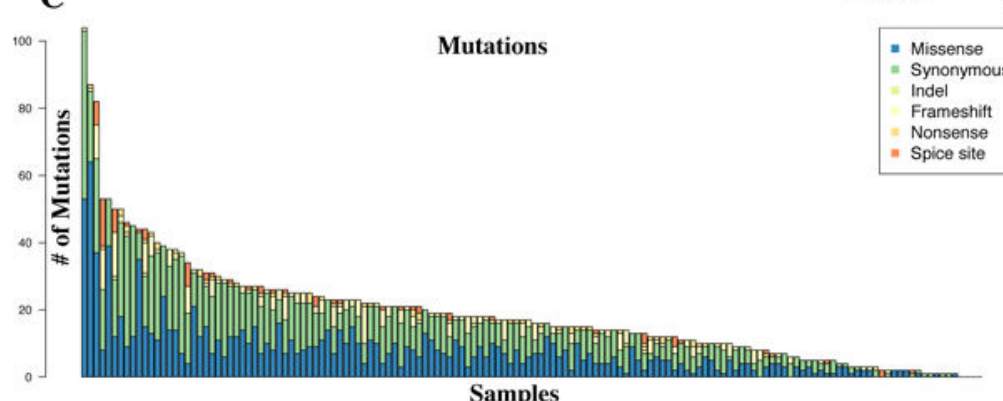

D
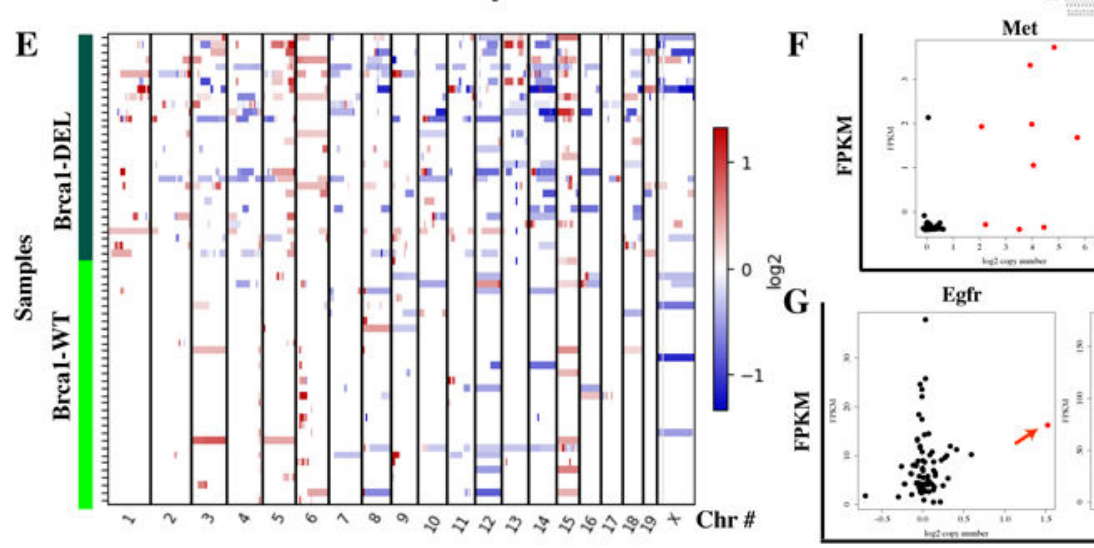

Figure 1.

Establishment of TNBC mouse models that recapitulate many aspects of human TNBC including heterogeneous genomic alterations A) H\&E and IHC staining of ER, PR and HER2 for tumors developed in GEMM models. B) Transcriptional classification using AIMS reveals endogenous tumors developed in the mouse models are most similar to basallike breast cancer. C) Endogenous mouse tumors show a range of mutations. D) Mutational signature for each Brcal genotype. The six boxes show the relative frequency of each of the six substitution types, and within each box are the 16 substitution contexts depending on the $5^{\prime}$ and $3^{\prime}$ base pair context. E) Global CNA profile shows heterogeneous amplifications and deletions among different tumors. F) Recurrent amplification at the Met and Yap1 loci in the mouse tumors and their corresponding mRNA levels. Amplified samples are in red. G) Individual tumors with Egfr or Fgfr2 amplification express higher mRNA levels (expressed as Fragments Per Kilobase Million or PRKM) of Egfr or Fgfr2, respectively, while a tumor 
with Pten deletion shows low Pten transcript level. Arrows indicating amplified or deleted samples are in red. 
A

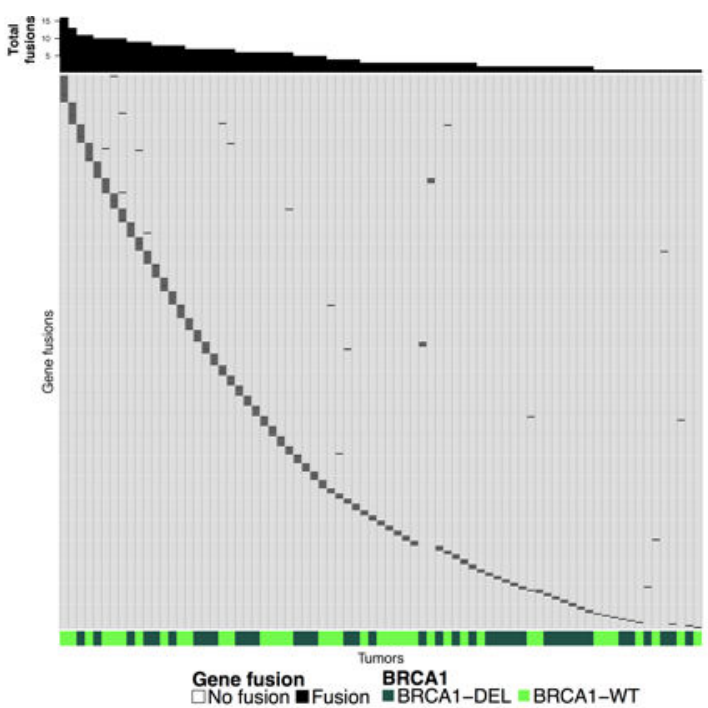

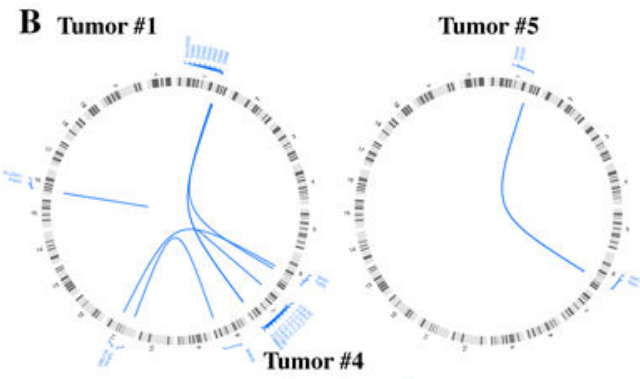

C
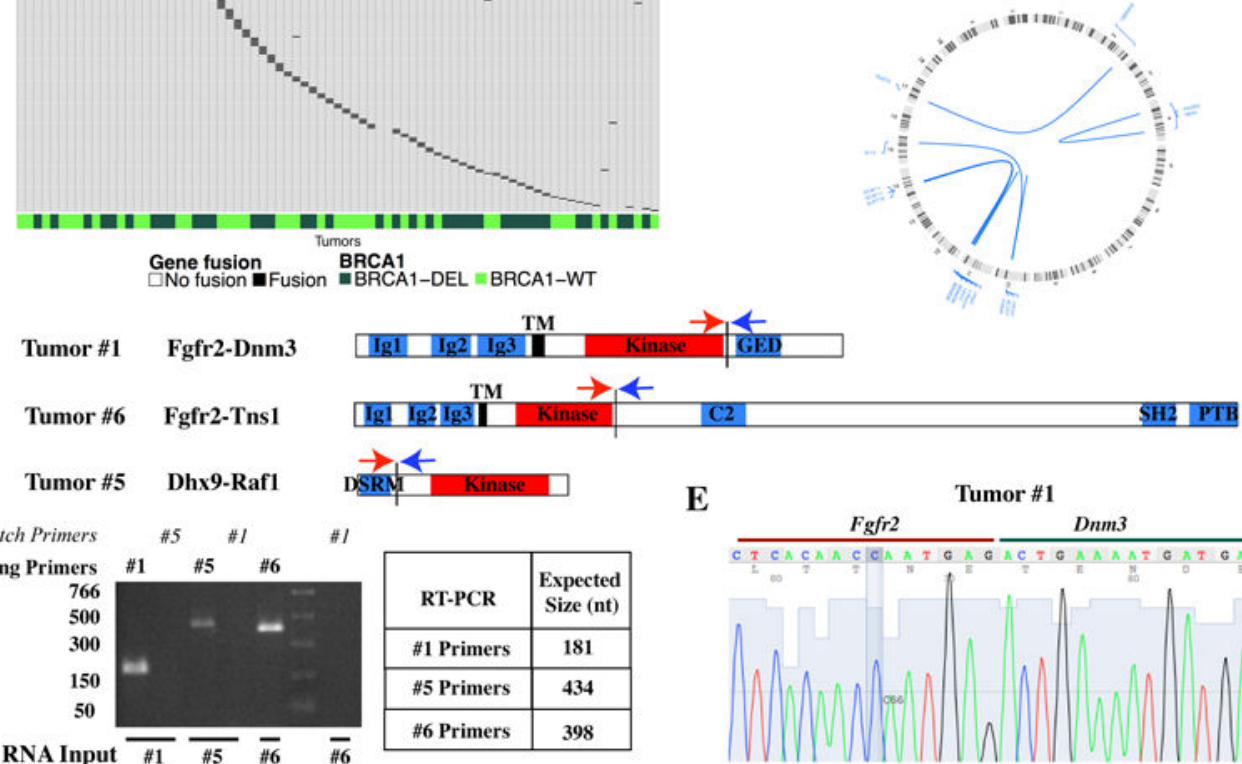

E

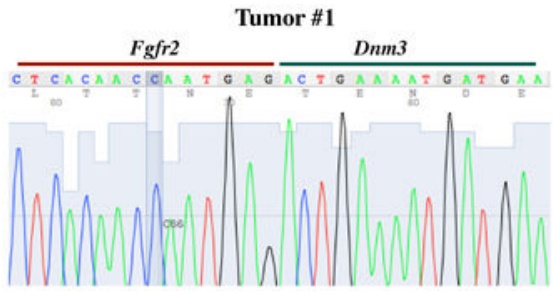

Figure 2.

Next generation sequencing of the endogenous TNBC tumors from GEMM models identifies multiple chromosomal rearrangement events. A) An overall view of fusion events in mouse breast tumors. B) Representative Circos plots show different numbers and pattern in chromosomal translocations among different tumors. C) Domain structure of Fgfr2-, Braf- and Raf1-fusion kinases. D) RT-PCR and E) Sanger sequencing confirming the presence of Fgfr2-Dnm3, Fgfr2-Tns1, and Dhx 9-Raf1 fusion transcripts in each of the spontaneous tumors. 


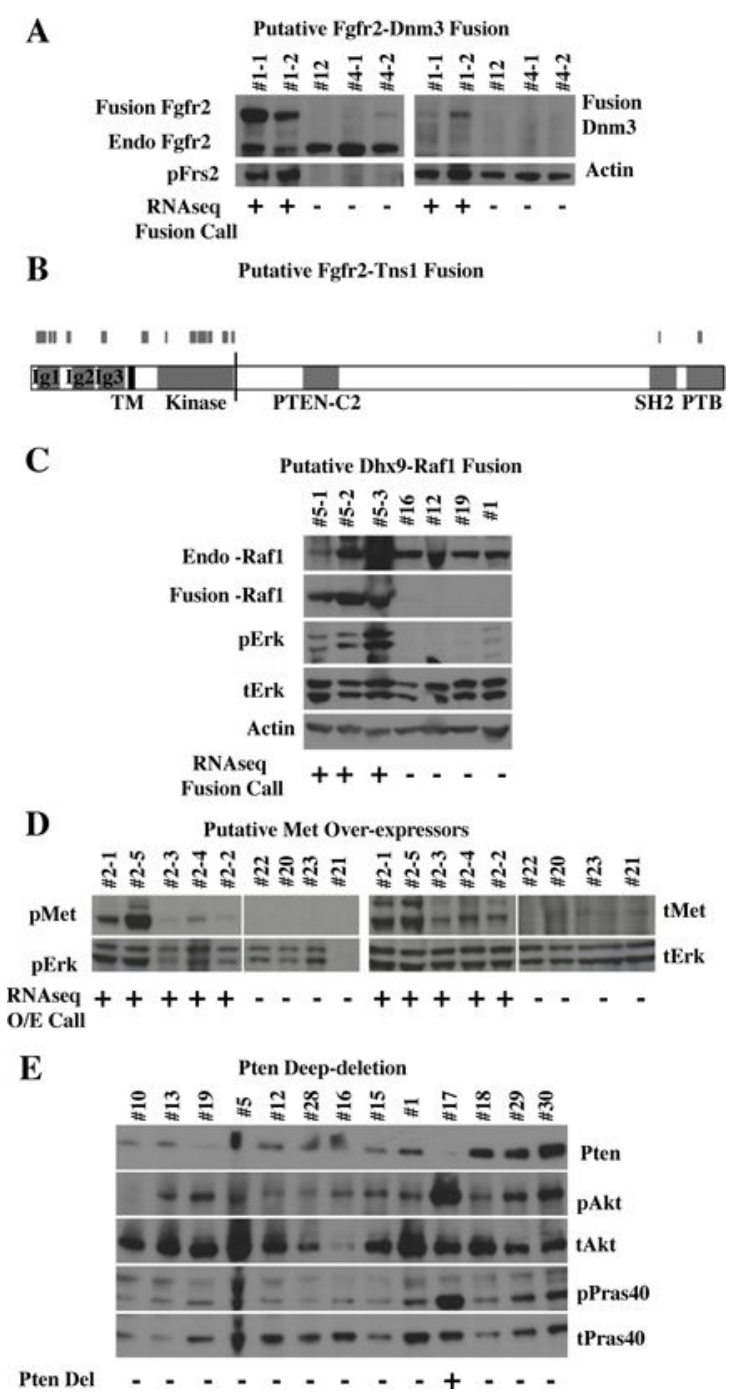

Figure 3 .

Western blotting confirming the presence of fusion proteins in tumor lysates and showing increased signaling pathway activation in respective tumors. A) Tumor \#1 series express Fgfr2-Dnm3 fusion proteins at expected molecular weight (left). At the same molecular weight, its fusion partner Dnm3 is also detected. Fgfr2-Dnm3 expression coincides with enhanced FRS2 phosphorylation. B) Detection of Fgfr2-Tns1 in tumor \#6 by mass spectrometry (MS) upon Fgfr2-immunoprecipitation. Short gray blocks show the locations of peptides identified by MS. C) Tumor \#3 series express Dhx9-Raf1 fusion proteins at the expected molecular weight with increased pERK signals. D) Tumor \#2 express high level of cMet at the protein levels, which coincide with increased Met phosphorylation. E) Tumor \#17 that harbors bi-allelic Pten deletion expresses undetectable Pten at the protein levels, and high Akt- and Pras40-phosphorylation. 


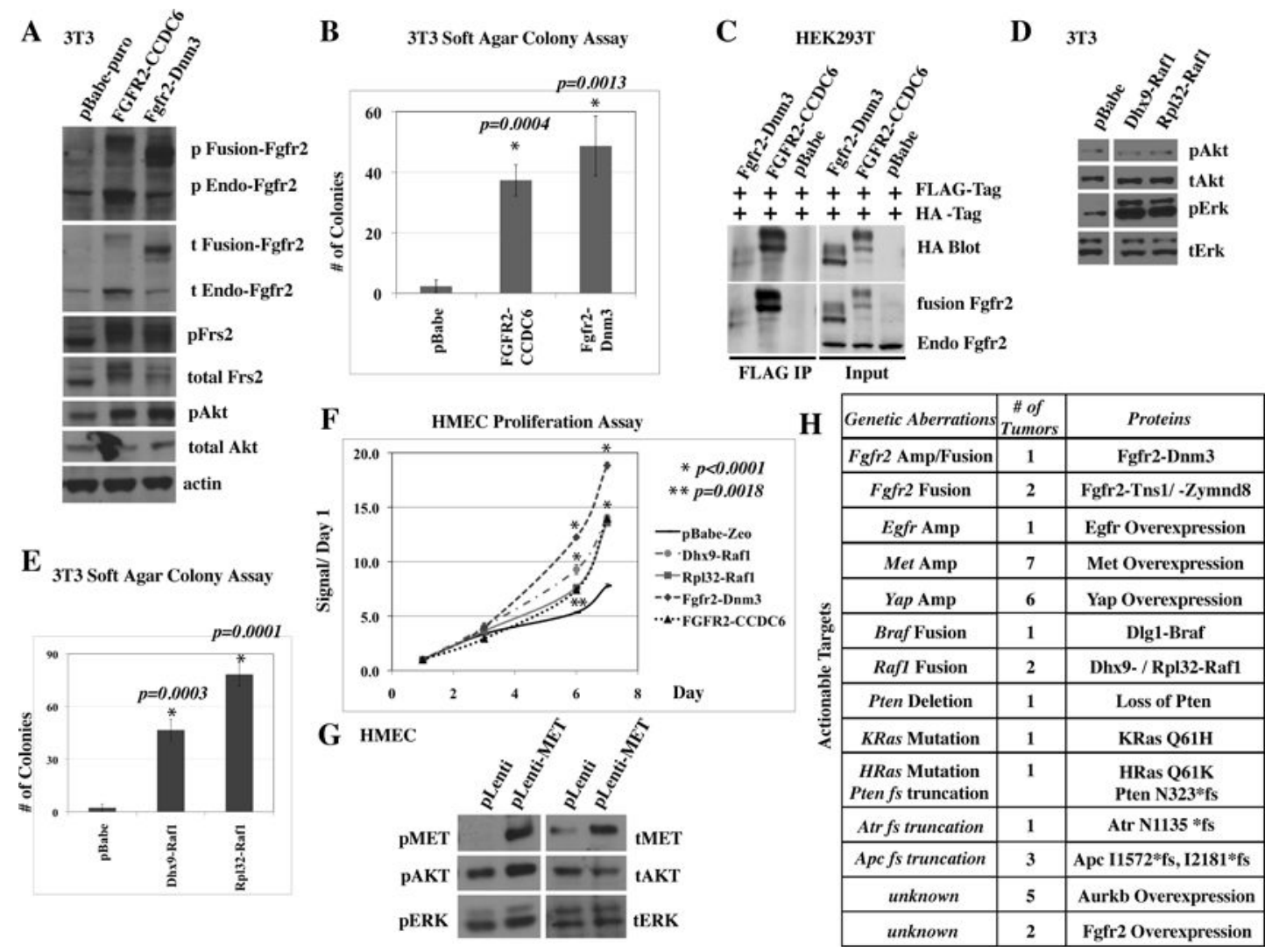

Figure 4.

Kinase fusions function as oncogenic drivers via enhanced activation of the MAPK and/or PI3K pathways. A) Expression of FGFR2-CCDC6 or Fgfr2-Dnm3 results in increased Fgfr2, Frs2 and downstream Akt phosphorylation in NIH3T3 cells. B) FGFR2-CCDC6 and Fgfr2-Dnm3 confer soft-agar colony formation capacity in NIH3T3 cells. C) Both Fgfr2Dnm3 and FGFR2-CCDC6 dimerize/oligomerize in a ligand-independent manner with fusion kinases but not with endogenous Fgfr2. D) Expressing of Dhx9-Raf1 or Rpl32-Raf1 fusion kinases significantly enhances the MAPK pathway in NIH3T3 cells. E) Dhx9-Raf1 and Rpl32-Raf1 fusion kinases confer soft-agar colony formation ability. F) Expression of FGFR2-CCDC6, Fgfr2-Dnm3, Dhx9-Raf1 or Rpl32-Raf1 increases HMEC proliferation. G) MET overexpression in HMECs induces spontaneous MET phosphorylation. H) Potential oncogenic driver events identified through combined WES and RNAseq analyses. 
A

Tumor \#1 (Fgfr2-Dnm3): Brca1-deficient
\begin{tabular}{|c|c|c|c|}
\hline Tumor \#1 & Solvent & Dose & Route \\
\hline BKM120 & $0.5 \%$ Methylcellulose/0.5\% Tween 80 & $30 \mathrm{mg} / \mathrm{Kg}$ & Oral \\
\hline BGJ398 & PEG300:D5W=2:1 & $30 \mathrm{mg} / \mathrm{Kg}$ & Oral \\
\hline Olaparib & DMSO & $100 \mathrm{mg} / \mathrm{Kg}$ & I.P. \\
\hline $\begin{array}{l}\text { BGJ398 } \\
\text { Olaparib }\end{array}$ & PEG300:D5W=2:1 & $30 \mathrm{mg} / \mathrm{Kg} \mathrm{BGJ}$ & Oral \\
\hline Crizotinib & $\mathbf{0 . 5 \%}$ Methylcellulose/0.5\% Tween 80 & $50 \mathrm{mg} / \mathrm{Kg}$ & Oral \\
\hline
\end{tabular}

C

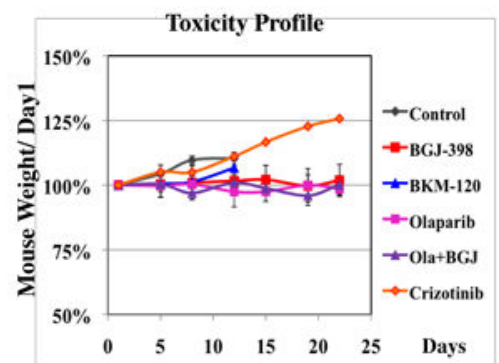

E

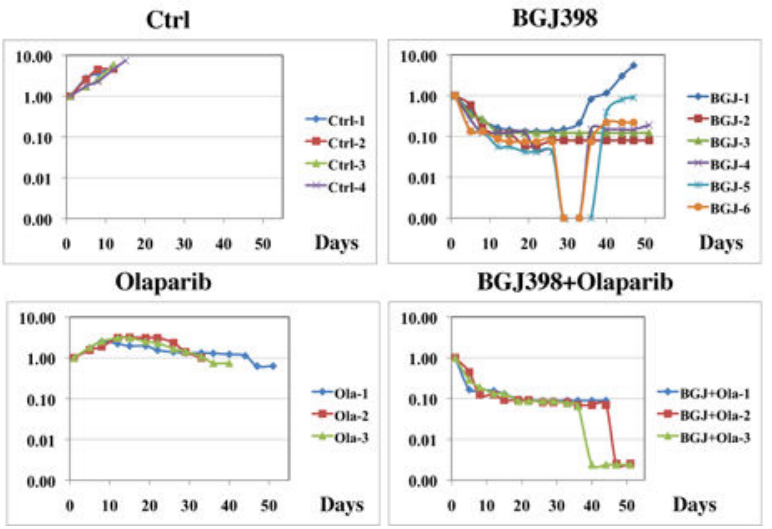

B

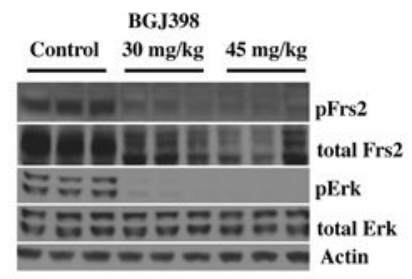

D

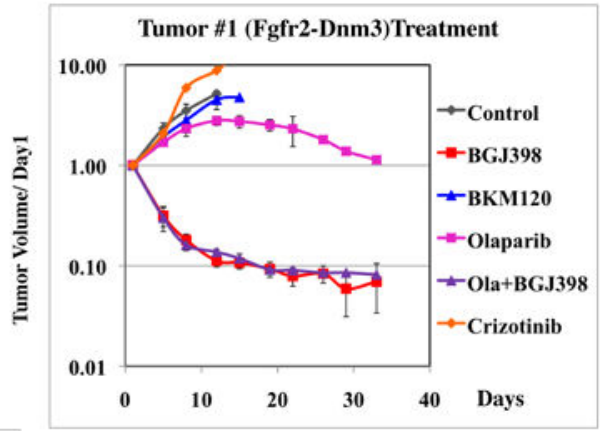

$\mathbf{F}$

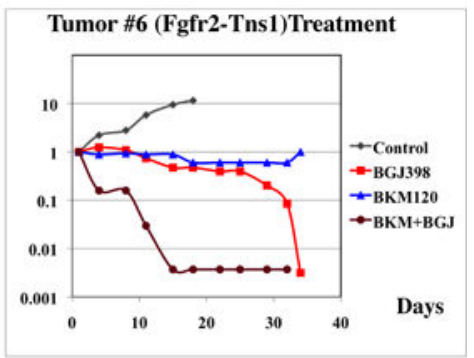

Figure 5.

Targeting Fgfr2-fusion containing tumors with the FGFR-inhibitor BGJ398 results in complete response. A) Treatment designs for tumor \#1 that is Brcal-deficient and harbors Fgfr2-Dnm3 translocation. B) In vivo dosing of BGJ398 at 30mg/kg achieves target inhibition measured by pFrs2 and pErk levels. C) BGJ398 alone, or in combination with Olaparib does not cause general toxicity in nude mice. D) BGJ398 alone or in combination with Olaparib results in complete tumor remission. E) Spider plot showing tumor responses to each treatment over long duration. F) BGJ398 alone results in slow tumor regression while BKM120 and BGJ398 causes fast tumor remission. 
A

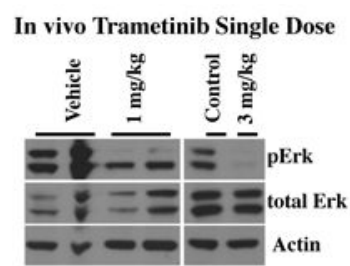

C
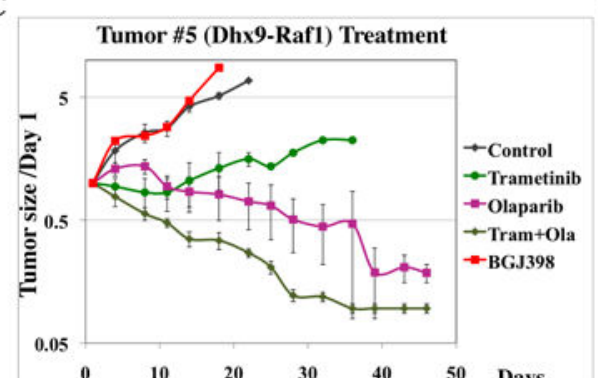

\begin{tabular}{|c|c|c|}
\hline Tumor 1614 & Solvent & Dose \\
\hline Trametinib & $10 \%$ Cremophor EL/10\% PEG400 & $3 \mathrm{mg} / \mathrm{Kg}$ \\
\hline Olaparib & DMSO & $25 \mathrm{mg} / \mathrm{Kg}$ \\
\hline $\begin{array}{c}\text { Trametibnib } \\
\text { Olaparib }\end{array}$ & $\begin{array}{c}10 \% \text { Cremophor EL/10\% PEG400 } \\
\text { DMSO }\end{array}$ & $\begin{array}{c}3 \mathrm{mg} / \mathrm{Kg} \mathrm{BKM} \\
\mathrm{mg} / \mathrm{Kg} \text { Ola }\end{array}$ \\
\hline
\end{tabular}

D



E

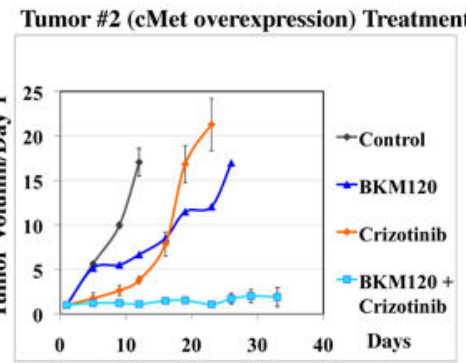

F

Tumor \#2 (cMet-Overexpressing): Brcal-proficient

\begin{tabular}{|c|c|c|c|}
\hline Tumor 1259 & Solvent & Dose & Route \\
\hline Crizotinb & $0.5 \%$ Methylcellulose/0.5\% Tween 80 & $50 \mathrm{mg} / \mathrm{Kg}$ & Oral \\
\hline BKM120 & $0.5 \%$ Methylcellulose $/ 0.5 \%$ Tween 80 & $30 \mathrm{mg} / \mathrm{Kg}$ & Oral \\
\hline Crizotinib & $0.5 \%$ Methylcellulose/0.5\% Tween 80 & $25 \mathrm{mg} / \mathrm{Kg} \mathrm{Criz}$ & Oral \\
BKM120 & $25 \mathrm{mg} / \mathrm{Kg} \mathrm{BKM}$ & Oral \\
\hline
\end{tabular}

Figure 6.

Targeting Dhx9-Raf1 and cMet with MEK- and MET-inhibitor, respectively, result in tumor regression or delayed progression. A) MEK inhibitor Trametinib (GSK1120212) achieves target inhibition at $3 \mathrm{mg} / \mathrm{kg}$. B) Treatment designs for Brca1-deficient tumor \#5 harboring Dhx 9-Raf1 translocation. C) Trametinib alone delays tumor progression while Trametinib and Olaparib result in complete tumor remission. D) At 50mg/kg, Crizotinib inhibits MET phosphorylation and downstream signaling pathway activation. E) Targeting Metoverexpressing endogenous tumor with MET-inhibitor delays tumor progression, and combination therapy results in stable disease. F) Treatment designs for tumor \#2 that is Brca1-WT and overexpresses cMET. 
A

\begin{tabular}{|l|c|c|c|}
\hline \multicolumn{1}{|c|}{ Gene Name } & TCGA & TCGA & METABRIC \\
& TNBC (82) & ALL BC(816 & $(2509)$ \\
\hline KRAS: Mut \& Amp & $11 \%$ & $3 \%$ & $2.6 \%$ \\
\hline BRAF: Mut \& Amp & $5 \%$ & $2.3 \%$ & $2.3 \%$ \\
\hline RAF1: Mut \& Amp & $4 \%$ & $2.2 \%$ & $1 \%$ \\
\hline EGFR:Mut \& Amp \& Exp>2 & $17 \%$ & $6 \%$ & $9 \%$ \\
\hline MET: Mut \& Amp \& Exp>2 & $27 \%$ & $7 \%$ & $4 \%$ \\
\hline FGFR1: Mut \& Amp \& Exp>2 & $12 \%$ & $17 \%$ & $14 \%$ \\
\hline FGFR2: Mut \& Amp \& Exp>2 & $16 \%$ & $8 \%$ & $4 \%$ \\
\hline FGFR3: Mut \& Amp \& Exp>2 & $4 \%$ & $3 \%$ & $1.8 \%$ \\
\hline YAP1: Amp \& Gain & $29 \%$ & $12 \%$ & $4 \%$ \\
\hline PIK3CA: Amp \& Mut=E454 Mut=H1047 & $17 \%$ & $20 \%$ & $22 \%$ \\
\hline PTEN: HOMDEL \& HETLOSS & $49 \%$ & $29 \%$ & $17 \%$ \\
\hline INPP4B: HETLOSS & $46 \%$ & $26 \%$ & $13 \%$ \\
\hline \multicolumn{1}{|c|}{ Overall } & $93 \%$ & $72 \%$ & $56 \%$ \\
\hline
\end{tabular}

Location and Frequency of Mutations in Breast Cancer



B

Amp $\begin{array}{lll}\text { Amp } & 1.7 \%\end{array}$

C



$\mathbf{E}$

MET Amp/OE FGFR fusion FGFR Amp/OE EGFR Amp/OF



Figure 7.

Multiple, different genetic aberrations lead to common elevated MAPK and/or PI3K

pathway activation in human breast cancer patients. A) Frequent genetic alterations that are known to activate the MAPK and/or PI3K pathways in TNBC patients (CbioPortal TCGA data). B) Frequency of amplification at the FGFR1, FGFR2 and FGF19 loci. C) Genomic amplification of FGFR1 and FGFR2 results in increased mRNA expression levels. D) Location and frequency of mutations found in FGFR 1 and FGFR2 in breast cancer. E) A model showing multiple genetic aberrations in TNBC leading to the activation of MAPK and/or PI3K pathways. 